\title{
Measuring understanding of nanoscience and nanotechnology: Development and validation of the nano-knowledge instrument (NanoKI)
}

\author{
Konrad J. Schönborn, Gunnar E. Höst, \& Karljohan E. Lundin Palmerius
}

\section{Postprint}

N.B.: When citing this work, please cite the original article.

Original Publication:

Schönborn, K.J., Höst, G.E., \& Lundin Palmerius, K.E. (2015). Measuring understanding of nanoscience and nanotechnology: development and validation of the nano-knowledge instrument (NanoKI). Chemistry Education Research and Practice, 16(2), 346-354

http://dx.doi.org/10.1039/C4RP00241E

Copyright: Royal Society of Chemistry 


\title{
Measuring understanding of nanoscience and nanotechnology: Development and validation of the nano-knowledge instrument (NanoKI)
}

\author{
K. J. Schönborn ${ }^{a *}$, G. E. Höst ${ }^{a}$ and K. E. Lundin Palmerius ${ }^{a}$
}

As the application of nanotechnology in everyday life impacts society, it becomes critical for citizens to have a scientific basis upon which to judge their perceived hopes and fears of 'nano'. Although multiple instruments have been designed for assessing attitudinal and affective aspects of nano, surprisingly little work has focused on developing tools to evaluate the conceptual knowledge dimension of public understanding. This article reports the validation of an instrument designed to measure conceptual knowledge of nanoscience and nanotechnology. A sample of 302 participants responded to a 28 -item questionnaire designed around core nano-concepts. Factor analysis revealed a single latent variable representing the construct of nano-knowledge. Cronbach's alpha was 0.91 indicating a high internal consistency of the questionnaire items. The mean test score was 15.3 out of 28 (54.5\%) with item difficulty indices ranging from 0.19 to 0.89 . Obtained item discrimination values indicate a high discriminatory power of the instrument. Taken together, the psychometric properties of the Nano-Knowledge Instrument (NanoKI) suggest that it is a valid and reliable tool for measuring nano-related knowledge. Preliminary qualitative observations of citizens' incorrect and correct response patterns to the questionnaire indicate potential conceptual challenges surrounding relative size of the nanoscale, random motion of nano-objects, and nanoscale interactions, although these are hypotheses that require future investigation. Application of the NanoKI could support efforts directed to an agenda for evaluating and designing science communication and education initiatives for promoting understanding of nano.

\section{Introduction}

Nanoscience is rapidly becoming a revolutionary and core component of research interconnected with multiple areas of scientific endeavour (Roco, 2003; Whitesides, 2005). Absorption of real practical applications of nanoscience and nanotechnology into the daily life of citizens is underway (Sealy, 2006). While the implications of 'nano' continue to emerge in manifestations of cutting-edge nanomaterials and nanotherapeutics, many contemporary scholars (e.g., Roco and Bainbridge, 2005; Burri and Bellucci, 2008), deem it crucial that the international public be actively involved in discussion, decisions and policy associated with nano. In this regard, Laherto (2010) and Gilbert and Lin (2013) advocate the urgent implementation of a nano-education vision that not only caters to formal academic demands necessary for accruing nanocompetent workers, but also considers informal public dimensions in evoking the societal implications of nanoscience.
This need is succinctly captured in Laherto's (2010) assertion that, "all citizens will soon need some kind of 'nano-literacy' in order to navigate important science-based issues related to their everyday lives and society" (p. 161).

The nano-revolution is playing out in the convergence of nano with new technological innovation. The inevitable impact of nano on society requires international citizens to be able to make scientifically-based judgments about associated perceived benefits, risks, hopes and fears (Roco and Bainbridge, 2005; Waldron et al., 2006; Schütz and Wiedemann, 2008). This premise is also closely intertwined with the mandate of science education in disciplines such as chemistry and biology education where it is becoming increasingly necessary to connect core discipline based concepts to the nanoscale in preparation of the modern learner (Muniz and Oliver-Hoyo, 2014; Orgill and Wood, 2014; Schönborn et al., 2014).

With respect to the role of nano-related knowledge in chemistry education, specific connections can be made between 
core discipline-based chemistry concepts and nanoscience. For example, as part of a recent special issue in the Journal of Nano Education, Orgill and Wood (2014) review and unpack specific chemistry contributions to nano education. In the same issue, St. Angelo (2014) demonstrates the integration of traditional chemistry curricula with nano, which include specific links between inorganic chemistry concepts such as hard sphere packing and unit cells to the self-assembly of nanoparticles and formation of monolayers. Furthermore, Muniz and Oliver-Hoyo (2014) have recently argued that since nano-related principles share a close relationship with core chemical concepts, more research is required to "explicitly connect these core concepts in a meaningful way to the nanoscale" (p. 807). In pursuit of such links, these authors have developed instructional undergraduate materials that explicitly connect core chemical concepts such as oscillatory behaviour and classical molecular mechanics to those at the nanoscale. In further support of the premise that nano can serve as a "unifying idea" in chemistry education, Jones et al. (2015) advocate that chemical concepts such as molecular bonding and molecular motion are connected to developing an understanding of self-assembly at the nanoscale. Similarly, Höst et al. (2013) demonstrate the importance of integrating several chemical concepts such as noncovalent interactions and complementarity in conceptualising the nanotechnological implications of selfassembly. Lastly, our own work (e.g. Schönborn et al., 2014) has also discerned how school discipline-based chemistry concepts such as intermolecular forces and binding specificity map onto an understanding of nanotechnological scenarios underlying the risk and benefit notions of nano-toxicity and nano-therapy, respectively. Overall, the studies above indicate that the communication of nanoscience can serve as a meaningful conceptual medium for connecting and learning in discipline-based chemistry education.

Perceptions and knowledge are integral to any public understanding of science (Cobb and Macoubrie, 2004) as well as fundamental to learning science in formal school and undergraduate settings. In this regard, the last decade has seen a rapid increase in the design of valid instruments for diagnosing citizens' and learners' understanding of scientific phenomena across both informal and formal learning contexts. Along with the rise in such test development work, multiple tests for probing a single scientific area have often been developed and validated. For example, Christensen et al. (2010) have developed an instrument to examine public understanding of basic genetics concepts among Americans, while Smith et al. (2008) have designed and validated a 25-item concept assessment to measure student understanding of genetics. In chemistry, various diagnostic instruments have been designed and validated to assess conceptual components of chemistry including covalent bonding and structure (Peterson et al.,1989) and a recent assessment tool developed to measure chemistry specific visual-perceptual skills (Oliver-Hoyo and Sloan, 2014). Furthermore, Shwartz et al. (2006) have developed and validated a multi-dimensional concept assessment to measure students' chemical literacy, wherein the authors also highlight the importance of applying such instruments to fostering the understanding of chemical concepts among the public.

Apart from the above diagnostic tools offered to probe constructs such as "genetic literacy" and "chemical literacy", the emerging notion of "nano literacy" has also witnessed the development and testing of multiple instruments focused on assessing attitudinal, affective and perception dimensions (e.g., Bainbridge, 2002; Cobb and Macoubrie, 2004; Siegrist et al., 2007; Lin et al., 2013). However, very little impetus has been on validating psychometric tools focused on the conceptual knowledge dimension of understanding nano per se: knowledge that deals with the scientific principles of nano. According to Dyehouse et al. (2008), one possible reason for this dearth in instrument design is that it is very challenging to construct items that are appropriate measures of nano-related understanding. Not only is nano interdisciplinary, it is also difficult to define what it is exactly that is representative of fundamental nano-knowledge.

Given the above, our investigation of the literature has discerned very few contributions associated with any formal measurement and validation of conceptual aspects of nanoknowledge. Lee et al. (2005) have explored interactions between cognitive and affective influences on public attitudes to nano, where six dichotomous items, including, "a nanometer is a billionth of a meter" and, "nanotechnology allows scientists to arrange molecules in a way that does not occur in nature" were deployed to measure knowledge about nanotechnology as a cognitive variable. Responses delivered a mean of $65 \%$ correct responses from 706 telephonic survey participants in the United States (also see Scheufele et al., 2009). A recent public knowledge instrument has been developed by Lin and colleagues (2013) to assess Taiwanese citizens' nanotechnology knowledge. The content and subscales of the native-language instrument was based on the work of Stevens et al. (2009), where essential links for understanding nano from an educational perspective were forged. Administering the multiple-choice test to 209 adults revealed that approximately $70 \%$ of the Taiwanese respondents delivered inadequate nanorelated understanding.

Measuring nano-related knowledge can inform policy makers in the implementation of effective science communication channels for evaluating and engendering public understanding of nano. In turn, this goes hand-in-hand with designing educational resources and instructional interventions for learning and teaching nanoscience, as well as for using nano as a context to teach core discipline-based concepts in chemistry education. However, the relative lack of valid instruments may impede further progress in these important areas. Therefore, we identify the design and development of valid and reliable tests as a crucial need for nanoeducation and scientific literacy at large. Hence, the aim of this study was to design and validate an instrument for measuring conceptual knowledge central to nanoscience and nanotechnology.

\section{Methods}




\section{Instrument design and development}

Design and development of the instrument spanned for a period of one year. The process commenced with a thorough literature review focused on identifying core concepts, principles and knowledge regarded as fundamental to understanding and reasoning about nano from an educational point of view. This took the form of identifying and consulting approximately 120 contributions in the literature that contained, alluded to, or focused on nano-related education content. Examples of noteworthy contributions in this regard included Batt et al. (2008), Jones et al., (2013), Lee et al. (2005), Lin et al. (2013), Scheufele et al. (2009) and Stevens et al. (2009)). An inductive literature analysis (e.g. Suikkala and Leino-Kilpi, 2001) was performed to identify themes in the literature set (e.g. Jones et al., 2011) that represented salient features of content related to communicating "nano-knowledge". As core concepts such as relative size, relative scale, area, volume, nanometer, adhesion, intermolecular forces, relative forces, gravity, binding specificity, and random motion, emerged prevalently, they naturally converged into separate umbrella properties and descriptions that captured nano-related knowledge. Inductive synthesis of the nano-related education literature resulted in the emergence of six themes namely, i) sizes of nano-objects, ii) forces and interactions between nano-objects, iii) dynamics and movement of nano-objects, iv) surface area:volume relationship, v) implications of shifting scale, and vi) nanotechnological applications. Further analysis of the six themes resulted in the respective merging of the closely related themes (ii) and (iii) as well as (v) and (vi), resulting in the following four overarching themes garnered from the literature:

1. The nanoscale and the relative size of nano-objects,

2. Dynamics, forces and interactions between objects at the nanoscale,

3. Consequences of the surface area:volume relationship of objects at different scales,

4. Implications of nano-properties for developing nanotechnologies.

The above emergent perspectives are interrelated and also represent the interdisciplinary and convergent nature of nano (e.g., see Roco, 2003) and together, represent one conceptual baseline necessary for eliciting a basic understanding of nano. It should be noted, however, that while an inductive content analysis approach can serve as an effective means to identify prevalent themes in literature content, the method does not necessarily expose all possible thematic structures inherent in a particular literature set (Suikkala and Leino-Kilpi, 2001). Guided by the four themes identified during the analysis, we first collated any existing items in the literature that were already closely related to the themes (e.g. Lee et al., 2005), and modified these item candidates into propositional statements (e.g. "a nanometer is a billionth of a meter" (Theme 1) and "nanotechnology allows scientists to arrange molecules in a way that does not occur in nature" (Theme 4)). We also developed an inventory of our own propositional statements related to the four themes . The set of propositional statements was then transformed into conceptual "true or false" utterances, which constituted a first draft of approximately 40 nanoknowledge test items.

Content validity of the test was established in three stages. First, specialists in science education research, molecular science, and public understanding of science discussed the items with the authors. This resulted in comments and suggestions for revising items. For example, the item "nanoobjects present in a liquid are in constant motion" (Theme 2) was revised to, "objects at the nanoscale are kept in random motion by continuous collisions with other particles". As part of this process. several redundant items were also eliminated from the test. Second, the semantics and syntax of the remaining items were carefully analysed and modified to remove any residual ambiguities. Third, as a measure of face validity, the readability and interpretation of the items were piloted and discussed with four adults with no background knowledge in nano-related areas. The test development process culminated in a final 28-item Nano-Knowledge Instrument (NanoKI), presented in the Appendix. Items 1-7 pertained to Theme 1 , items 8-14 to Theme 2 , items $15-18$ to Theme 3, and items 19-28 to Theme 4. A "True", "False" and "Don't know" answer category system was used for the test. The latter category was included to avoid forcing prospective test participants into guessing or responding correctly or incorrectly when they were not confident in their knowledge enough to commit to an answer (e.g., Carey et al., 1997; May and Pfäfflin, 2002; Winzenberg et al., 2003). Fourteen of the 28 statements are true $(\mathrm{T})$ and fourteen are false $(\mathrm{F})$, respectively (see Appendix).

\section{Data collection procedure}

The procedure for collecting data was designed with the aim of allowing the developed instrument to be validated by investigating its psychometric properties and its underlying conceptual structure. With this aim, a target sample size of 300 participants was set. In support of this planned sample size, statistical literature has recommended sample sizes of at least 300 for studying reliability (Nunnally and Bernstein, 1994), and for factor analysis (Tabachnick and Fidell, 2007).

Given that the instrument is intended for use with respondents from a range of backgrounds and levels of conceptual preknowledge, a broad sampling strategy was employed. It consisted of using social media platforms consisting of Twitter, Facebook and various Blogs to attract members of the public from around the world to respond to the NanoKI. In turn, potential international respondents were targeted through their engagement with these social media where we searched for communities registered within these platforms that were related to popular science, technology and science education, educational providers, libraries, science centres, science TV shows, newspapers, magazines, governmental agencies, and museums to expose the test to. Invitations to answer the questionnaire were sent out as posts and messages. For example, by asking a society that works to promote public access to science to include information about 
the study in one of their social network "feeds", any person that followed the digital feed would be potentially exposed to the study invitation. For practical and ethical reasons it was decided against recording the precise geographical distribution of the respondents. The reason was twofold; first, asking public respondents about their nationality might decrease their willingness to participate, and second, exploiting technological means of tracking the location of responses without participants' consent was considered unethical. Although we have no tracking of the precise geographic distribution of the respondents, we do know that the participants represent an international sample since the sampling strategy covered social media platforms emanating from at least 20 countries around the world. This was also inadvertently further supported by respondents' answers generated from an optional final openended question that asked for any general comments about the test as a whole, where some participants spontaneously provided information that revealed their geographical location. In addition, based on the unique demographic information obtained for each data point, it was unlikely that any participant retook the test.

The NanoKI was administered in the form of a web-based questionnaire. Data collection was conducted over three months and participation was anonymous. Informed consent was gathered by informing potential participants about the aim of the study and requiring a tick-box to be checked to commence the test. The respondents were first asked to state their gender and age, indicate their level of education, and to rate their interest in nanoscience and nanotechnology. Table 1 summarizes the characteristics of the sample of participants. Following the background questions, the 28 test items were presented on the screen one at a time, where participants were asked to, "Indicate whether each of the following statements are true or false, or indicate if you do not know", in the order that they appear in the Appendix.

[Location of Table 1 is about here]

\section{Data analysis}

The test was scored by assigning a value of 1 to correct responses (i.e. choosing "True" for a true statement, and viceversa) and a value of 0 to incorrect responses (i.e. choosing "True" for a false statement and vice-versa). Responses in the "Don't know" category were awarded a value of 0 (e.g., Winzenberg et al., 2003; Sütterlin et al., 2011). Summing correct responses corresponded to an individual possible total test score that ranged from 0 to 28 .

The test was validated using the following psychometric measures. Readability of the items was assessed using the Flesch-Kincaid measure. Item performance was analysed by calculating the proportion of correct answers (difficulty index) and the mean difference between high scorers and low scorers (discrimination index D) for each item. Inter-item correlations were calculated to detect any negative and low correlations. As measures of the impact of each item on the overall scale, corrected item-total correlations and Cronbach's alpha if item deleted were calculated. Reliability of the test was assessed from the overall Cronbach's alpha.

The construct validity of the test was investigated through factor analysis to discern any substructures in the data. The factor analysis procedure employed principal component analysis (PCA) to analyse the matrix of correlations. As recommended in the psychometric testing literature (Henson and Roberts, 2006), multiple criteria were used to deduce the number of factors to retain.

\section{Results and discussion}

A total of 302 individuals completed the questionnaire. Validation of the instrument and analysis of the generated responses are presented in the following sections.

\section{Validation of the NanoKI}

Analysis of the responses to the 28 items revealed a mean score of 15.3 ( $\mathrm{SD}=6.90$, median=16), corresponding to $54.5 \%$ correct responses. The participants' scores ranged from 0 to 28 . Completing the 28 items took the participants slightly less than 8 minutes on average ( $M=465$ s, SD=278 s). A Cronbach's alpha value of 0.907 was obtained for the test, which indicates a high internal consistency. Overall readability of the test corresponded to the typical reading level of a 16-18 year old (11.5th grade), according to the Flesch-Kincaid formula. Table 2 provides detailed item-by-item psychometric properties of the instrument (cf. Appendix).

\section{[Location of Table 2 is about here]}

As shown in Table 2, the item difficulty indexes ranged between 0.19 and 0.89 . Three quarters of the items had difficulty indexes in the range $0.25-0.75$, indicating that overall, the test is neither too easy nor too difficult. Discrimination values for all items except one (Q3) were above 0.4, indicating a good discriminatory power of the instrument (Doran, 1980).

The suitability of the individual items and internal consistency of the test was further ascertained through several measures. The correlation matrix showed that all item-item correlations were positive, with a mean value of 0.26 , indicating that the items are consistent without being redundant (Briggs and Cheek, 1986). In addition, corrected item-total correlations were above 0.30 , with the exception of Q3 and Q8 which were slightly below 0.3 . This indicates that the items correlate well with the total test score (Field, 2009). Finally, Cronbach's alpha was not improved by removing any of the 28 items (Table 2).

Factor analysis using Principal Component Analysis (PCA) was conducted on the 28 items. Suitability of applying PCA on the data was supported by a Kaiser-Meyer-Olkin measure of sampling adequacy above $0.6(0.917)$ and a significant result on Bartlett's test of sphericity $(\mathrm{p}<0.0005)$ (Tabachnick and Fidell, 2007). Six components with eigenvalues greater than 1 were extracted (Figure 1), explaining 29.3\% (eigenvalue 8.21), 5.6\% (eigenvalue 1.57), 4.8\% (eigenvalue 1.34), $4.0 \%$ (eigenvalue 
1.11), 3.8\% (eigenvalue 1.05), and 3.7\% (eigenvalue 1.03) of the variance, respectively.

[Location of Fig. 1 is about here]

Visual inspection of the scree plot (Figure 1) revealed a clear break after the first factor, indicating that only one factor should be retained. Subsequent Parallel Analysis indicated that the first two components are larger than the corresponding simulated eigenvalues calculated for a randomly generated data matrix of equal size ( 28 variables $\times 302$ respondents). Although Parallel Analysis indicated two latent variables in the data, factor analysis of binary variables may produce a spurious factor that reflects differences in difficulty between items (Green, 1983). Hence, a Minimum Average Partial test (Velicer et al., 2000) was performed which supported the scree analysis suggesting that one factor should be retained. Taken together, the structure of the data is consistent with a single latent variable. Given that all 28 items load onto this variable with factor loadings larger than 0.3 (Table 2), the latent variable was interpreted as a general "nano-knowledge" construct.

\section{Conceptual considerations arising from responses to the NanoKI}

Of the $45.5 \%$ of responses not coded as correct, $10.9 \%$ of responses were scientifically incorrect, while the remaining $34.6 \%$ were obtained from citizens not having any knowledge of the answer (i.e. "Don't know"). The most prevalent misunderstanding surrounded relative size of the nanoscale, mirroring current literature (e.g., Jones et al., 2013; Waldron et al., 2006; Delgado et al., 2015). Here, 40\% of responses incorrectly suggested that "A nanometer is 1000000 (1 million) times smaller than a meter" (False). In further relation to reasoning about relative size, $24 \%$ of the sample responded incorrectly to the item "The smaller an object is, the smaller its surface area compared to its volume" (False), and 17\% to "An animal cell is about 10 nanometers wide" (False). Fifty-six percent of the participants responded "Don't know" to the latter item.

When it came to ideas about random movement of nanoscale objects, $31 \%$ of respondents agreed that "Modified nanotubes will move directly towards their intended target after being introduced into the body" (False) and 49\% indicated that they did not know. This observation suggests erroneous anthropomorphic-related thinking (Klymkowsky and GarvinDoxas, 2008) possibly compounded by inappropriate "drug delivery" metaphors (Loeve et al., 2013). Furthermore, 19\% of respondents did not correctly express the fact that, "Objects at the nanoscale are kept in random motion by continuous collisions with other particles" (True), which perhaps indicates the conceptual demands of perceiving the "sticky", "shaky" and "bumpy" (Jones et al., 2013) properties of the nanoworld. Moreover, the observation that "Nanotubes spontaneously aggregate together into rope-like structures" (True) was unknown by $56 \%$ of the participants could indicate a lack of (albeit cognitively demanding) knowledge that emergent properties may arise from random molecular events (Höst et al., 2013).

In relation to nanoscale interactions, $61 \%$ of "Don't know" selections were attributed to the item, "A modified nanotube that has attached to its specific target will remain permanently bound" (False), while 56\% of such responses were obtained for, "Attractive forces between objects at the nanoscale become weaker when the contact surfaces of the objects have complementary shapes" (False). Overall, the observation that respondents often simply lacked any knowledge of the answer, is consistent with literature asserting that the public has little awareness and knowledge about nano-related phenomena (Waldron et al., 2006; Schütz and Wiedemann, 2008; Dyehouse et al., 2008).

Overall, the finding that a unidimensional construct underlies an instrument designed to cover four distinct conceptual themes warrants further research into the structural dimensions of nanoscience and nanotechnology knowledge. The integration of scientific disciplines that converge in the notion of "nanoscience and nanotechnology" may imply that any individual's knowledge of nano-related phenomena will by necessity, consist of interlinked concepts. It is also possible that the degree of correlation between conceptual knowledge across the four themes is a function of characteristics of the sample under study here.

\section{Methodological limitations of the study}

A limitation of the study is the frequent designation of nanotubes as the representative "nano-objects" of attention in the items, which may not capture the diversity of nanoscale matter in nanotechnology. However, the thorough procedure employed in the design of items should ensure that they cover important underlying nanoconcepts. In this context, it would be interesting to compare the item content and the performance of the few instruments that have been designed in different contexts to measure nano-knowledge (e.g., Lee et al., 2005; Lin et al., 2013). In this regard, given that the discipline of nanoscience is still relatively young, and that the idea of nano in itself is such an overarching construct, analysing correlations between the tests would offer a form of additional validation as to what concepts constitute nano-knowledge.

The sampling strategy used in the study is inherently associated with a form of self-selection bias in that $82 \%$ of the respondents that chose to participate were either interested or very interested in nano, and $69 \%$ of the respondents had a tertiary education qualification of more than three years. However, despite a potential bias in interest and education, the wide range of scores delivered by the respondents indicates that this bias did not skew distribution towards high levels of nanoknowledge. Therefore, the validation of the test should not be affected. It should be borne in mind, however, that the level of nano-knowledge measured in the present sample may not be immediately generalizable to any particular population. Such a generalisation would require a representative sampling strategy, and while seen as ideal, test development and validation often relies on non-random sampling techniques such as convenience 
sampling (e.g. Wells and Cartwright-Hatton, 2004; Vlachopoulos and Michailidou, 2006; Örücü and Demir, 2009; Lin et al., 2013). Assessing the understanding of core nanoscientific principles in defined populations is an important line of investigation. In this regard, NanoKI contributes to the development and availability of research tools for conducting such surveys.

\section{Conclusions}

Results of this study on an international sample of 302 volunteer respondents indicate that the 28 -item NanoKI is a valid and reliable test for measuring understanding of nanoscience and nanotechnology. The NanoKI shows high internal consistency, as revealed by an alpha value of 0.91 . Emergence of a single factor from the factor analysis indicates a unidimensional and homogeneous test instrument. The identified construct could be interpreted as a measure of basic nanoscience and nanotechnology knowledge.

In addition to serving as a valid measure of basic nanoknowledge, the pattern of correct and incorrect responses could also be a starting point for more detailed hypotheses about citizens' accurate and inaccurate scientific knowledge about nano. For instance, observations here indicate possible conceptual challenges surrounding relative size of the nanoscale, random motion, and nanoscale interactions, ideas that are also closely linked to core discipline-based concepts in formal chemistry education. Investigations of these hypotheses will constitute a future phase of this research project. In turn, such a pursuit could use NanoKI as a diagnostic educational tool for helping inform science teachers about the status of learners' nano-knowledge related to traditional chemistry concepts. The validation of NanoKI in an international context across a range of demographics, including age, gender, and educational level, implies that the instrument is appropriate for wide application. For example, using NanoKI to assess public understanding of nanoscience and nanotechnology could inform initiatives for government and regulatory policy, and for effective lines of communicating nano to the public. At the secondary school level, we are currently using NanoKI as part of investigating nano as a context for learning and teaching chemistry concepts.

It is a prerequisite for citizens to be able to make informed decisions about emerging technologies in any democracy. However, awareness and knowledge about nano remains fairly low. There is a need to spur the agenda on how best to develop understanding of nanoscience and nanotechnology. A tool for promoting and supporting this effort in informal as well as formal learning contexts is offered in the form of NanoKI.

\section{Acknowledgements}

This research was supported by the Swedish Research Council (Vetenskapsrådet, Grant 2011-5569). The authors thank Henry Fröcklin for technical assistance in construction of the electronic data-collection platform, colleagues at Linköping
University for valuable discussions in development of the NanoKI items, and the anonymous international respondents for participating in the study.

\section{Notes and references}

${ }^{a}$ Department of Science and Technology (ITN), Linköping University, Campus Norrköping, SE-601 74 Norrköping, Sweden.

* Corresponding author: Tel.: +46 113636 09, Fax: +46 113632 70, Email: konrad.schonborn@liu.se

Bainbridge W. S., (2002), Public Attitudes Toward Nanotechnology, J. Nanopart. Res., 4, 561-570.

Batt, C. A., Waldron, A. M. and Broadwater, N., (2008), Numbers, Scale and Symbols: The Public Understanding of Nanotechnology, $J$. Nanopart. Res., 10, 1141-1148.

Briggs S. R. and Cheek J. M., (1986), The Role of Factor Analysis in the Development and Evaluation of Personality Scales, J. Pers., 54, 106-148.

Burri R. V. and Bellucci S., (2008), Public Perception of Nanotechnology, J. Nanopart. Res., 10, 387-391.

Carey M. P., Morrison-Beedy D. and Johnson B. T., (1997), The HIVKnowledge Questionnaire: Development and Evaluation of a Reliable, Valid, and Practical Self-Administered Questionnaire, AIDS Behav., 1, 61-74.

Christensen, K. D., Jayaratne, T. E., Roberts, J. S., Kardia, S. L. R. and Petty, E. M., (2010), Understandings of Basic Genetics in The United States: Results from a National Survey of Black and White Men and Women, Public Health Genom., 13, 467-476.

Cobb M. D. and Macoubrie J., (2004), Public Perceptions about Nanotechnology: Risks, Benefits and Trust, J. Nanopart. Res., 6, 395-405

Delgado, C., Stevens, S. Y., Shin, N. and Krajcik, J. (2015), A Middle School Instructional Unit for Size and Scale Contextualized in Nanotechnology, Nanotechnol. Rev., 4, 51-69.

Doran R. L., (1980), Basic Measurement and Evaluation of Science Instruction, Washington, DC: NSTA Press.

Dyehouse M. A., Diefes-Dux H. A., Bennett D. E. and Imbrie P. K., (2008), Development of an Instrument to Measure Undergraduates' Nanotechnology Awareness, Exposure, Motivation, and Knowledge, J. Sci. Educ. Technol., 17, 500-510.

Field A., (2009), Discovering statistics using SPSS, London: SAGE.

Gilbert J. K. and Lin H.-S., (2013), How Might Adults Learn about New Science and Technology? The Case of Nanoscience and Nanotechnology, Int. J. Sci. Educ. Part B, 3, 267-292.

Green S. B., (1983), Identifiability of Spurious Factors Using Linear Factor Analysis with Binary Items, Appl. Psych. Meas., 7, 139-147.

Henson R. K. and Roberts J. K., (2006), Use of Exploratory Factor Analysis in Published Research: Common Errors and Some Comment on Improved Practice, Educ. Psychol. Meas., 66, 393416.

Höst G. E., Larsson C., Olson A. and Tibell L. A. E., (2013), Student Learning about Biomolecular Self-Assembly Using Two Different External Representations, CBE-Life Sci. Educ., 12, 471-482.

Jones, M. V., Coviello, N. and Tang, Y. K., (2011), International Entrepreneurship Research (1989-2009): A Domain Ontology and Thematic Analysis, J. Bus. Venturing, 26, 632-659. 
Jones, M. G., Gardner, G. E., Falvo, M. and Taylor, A., (2015), Precollege Nanotechnology Education: A Different Kind of Thinking, Nanotechnol. Rev.. 4, 117-127.

Jones M. G., Blonder R., Gardner G. E., Albe V., Falvo M. and Chevrier J., (2013), Nanotechnology and Nanoscale Science: Educational Challenges, Int. J. Sci. Educ., 35, 1490-1512.

Klymkowsky M. W. and Garvin-Doxas K., (2008), Recognizing Student Misconceptions through Ed's Tools and the Biology Concept Inventory, PLOS Biol., 6, e3.

Laherto A., (2010), An Analysis of the Educational Significance of Nanoscience and Nanotechnology in Scientific and Technological Literacy, Sci. Educ. Int., 21, 160-175.

Lee C.-J., Scheufele D. A. and Lewenstein B. V., (2005), Public Attitudes Toward Emerging Technologies: Examining the Interactive Effects of Cognitions and Affect on Public Attitudes Toward Nanotechnology, Sci. Commun., 27, 240-267.

Lin S.-F., Lin H.-S. and Wu Y.-Y., (2013), Validation and Exploration of Instruments for Assessing Public Knowledge of and Attitudes Toward Nanotechnology, J. Sci. Educ. Technol., 22, 548-559.

Loeve S., Vincent B. B. and Gazeau F., (2013), Nanomedicine Metaphors: From War to Care. Emergence of an Oecological Approach, Nano Today, 8, 560-565.

May T. W. and Pfäfflin M., (2002), The Efficacy of an Educational Treatment Program for Patients with Epilepsy (MOSES): Results of a Controlled, Randomized Study, Epilepsia, 43, 539-549.

Muniz, M. N., and Oliver-Hoyo, M. T., (2014), On the Use of Analogy to Connect Core Physical and Chemical Concepts to Those at the Nanoscale, Chem. Educ. Res. Pract., 15, 4, 807-823.

Nunnally J. C. and Bernstein I. H., (1994), Psychometric Theory, New York: McGraw-Hill.

Oliver-Hoyo, M. and Sloan, C., (2014), The Development of a VisualPerceptual Chemistry Specific (VPCS) Assessment Tool, J. Res. Sci. Teach., 51, 963-981.

Orgill, M. and Wood, S. A., (2014), Chemistry Contributions to Nanoscience and Nanotechnology Education: A Review of the Literature, J. Nano Educ., 6, 83-108.

Örücü, M. Ç. and Demir, A., (2009), Psychometric Evaluation of Perceived Stress Scale for Turkish University Students, Stress Health, 25, 103-109.

Peterson, R. F., Treagust, D. F. and Garnett, P., (1989), Development and Application of a Diagnostic Instrument to Evaluate Grade-11 and 12 Students' Concepts of Covalent Bonding and Structure following a Course of Instruction, J. Res. Sci. Teach., 26, 301-314.

Roco M. C., (2003), Nanotechnology: Convergence with Modern Biology and Medicine, Curr. Opin. Biotech., 14, 337-346.

Roco M. C. and Bainbridge W. S., (2005), Societal Implications of Nanoscience and Nanotechnology: Maximizing Human Benefit, $J$. Nanopart. Res., 7, 1-13.

Scheufele, D. A., Corley, E. A., Shih, T. J., Dalrymple, K. E. and Ho, S. S., (2009), Religious Beliefs and Public Attitudes Toward Nanotechnology in Europe and the United States. Nat. Nanotechnol., 4, 91-94.

Schönborn K. J., Höst G. E., Lundin Palmerius K. E. and Flint J., (2014), Development of an Interactive Immersion Environment for Engendering Understanding about Nanotechnology: Concept,
Construction, and Implementation, Int. J. Virt. Pers. Learn. Environ., 5, 40-56.

Schütz H. and Wiedemann P. M., (2008), Framing Effects on Risk Perception of Nanotechnology, Public. Underst. Sci., 17, 369-379.

Sealy C., (2006), Education, Education, Education, Nano Today, 1, 1.

Shwartz, Y., Ben-Zvi, R. and Hofstein, A., (2006), The use of Scientific Literacy Taxonomy for Assessing the Development of Chemical Literacy among High-School Students, Chem. Educ. Res. Pract., 7, 203-225.

Siegrist M., Keller C., Kastenholz H., Frey S. and Weik A., (2007), Laypeople's and Experts' Perception of Nanotechnology Hazards, Risk Anal., 27, 59-69.

Smith, M. K., Wood, W. B. and Knight, J. K., (2008), The Genetics Concept Assessment: A New Concept Inventory for Gauging Student Understanding of Genetics, CBE-Life Sci. Educ., 7, 422430.

St, Angelo, S. K., (2014), Bringing Nanoscience Into Traditional Physical and Inorganic Chemistry Courses, J. Nano Educ., 6, 132-138.

Stevens S., Sutherland L. and Krajcik J. S., (2009), The Big Ideas of Nanoscale Science \& Engineering: A Guidebook for Secondary Teachers. Arlington, VA: NSTA Press.

Suikkala, A. and Leino-Kilpi, H., (2001), Nursing Student-Patient Relationship: A Review of the Literature from 1984 to 1998, J. Adv. Nurs., 33, 42-50.

Sütterlin B., Brunner T. A. and Siegrist M., (2011), Who Puts the Most Energy into Energy Conservation? A Segmentation of Energy Consumers Based on Energy-Related Behavioral Characteristics, Energ. Policy, 39, 8137-8152.

Tabachnick B. G. and Fidell L. S., (2007), Using Multivariate Statistics, New York: Pearson.

Vlachopoulos, S. P. and Michailidou, S. (2006), Development and Initial Validation of a Measure of Autonomy, Competence, and Relatedness in Exercise: The Basic Psychological Needs in Exercise Scale, Meas. Phys. Educ. Exerc. Sci., 10, 179-201.

Velicer W. F., Eaton C. A. and Fava J. L., (2000), Construct Explication Through Factor or Component Analysis: A Review and Evaluation of Alternative Procedures for Determining the Number of Factors or Components, in Goffin R. D. and Helmes E. (ed.), Problems and Solutions in Human Assessment: Honouring Douglas N. Jackson at Seventy, Boston, MA: Kluwer, pp. 41-71.

Waldron A. M., Spencer D. and Batt C. A., (2006), The Current State of Public Understanding of Nanotechnology, J. Nanopart. Res., 8, 569-575.

Wells, A., and Cartwright-Hatton, S. (2004), A Short Form of the Metacognitions Questionnaire: Properties of the MCQ-30, Behav. Res. Ther., 42, 385-396.

Whitesides G. M., (2005), Nanoscience, Nanotechnology, and Chemistry, Small, 1, 172-179.

Winzenberg T. M., Oldenburg B., Frendin S. and Jones G., (2003), The Design of a Valid and Reliable Questionnaire to Measure Osteoporosis Knowledge in Women: The Osteoporosis Knowledge Assessment Tool (OKAT), BMC Musculoskel. Dis., 4, 1-7. 


\section{Appendix}

The Nano-Knowledge Instrument (NanoKI)

\begin{tabular}{|c|c|c|c|c|}
\hline Item & Statement & True & False & $\begin{array}{l}\text { Don't } \\
\text { know }\end{array}$ \\
\hline Q1 & A nanometer is 1000000 ( 1 million $)$ times smaller than a meter. & ○ & $\bullet$ & o \\
\hline Q2 & $\begin{array}{l}\text { Nanotechnology involves objects where the size in at least one direction is } \\
\text { between } 1 \text { and } 100 \text { nanometers. }\end{array}$ & $\bullet$ & $\circ$ & ○ \\
\hline Q3 & Objects at the nanoscale are just large enough to be visible to the naked eye. & ○ & $\bullet$ & $\circ$ \\
\hline Q4 & Objects at the nanoscale do not occupy any volume. & ० & $\bullet$ & $\circ$ \\
\hline Q5 & $\begin{array}{l}\text { Carbon nanotubes consist of carbon atoms bonded to each other in a regular } \\
\text { pattern. }\end{array}$ & $\bullet$ & $\circ$ & ○ \\
\hline Q6 & An animal cell is about 10 nanometers wide. & ○ & $\bullet$ & ○ \\
\hline Q7 & The length of a nanotube can be longer than the size of a human cell. & $\bullet$ & ○ & ○ \\
\hline Q8 & $\begin{array}{l}\text { Objects at the nanoscale are kept in random motion by continuous collisions with } \\
\text { other particles. }\end{array}$ & $\bullet$ & $\circ$ & ○ \\
\hline Q9 & $\begin{array}{l}\text { The strength of the forces between objects at the nanoscale plays a less dominant } \\
\text { role than gravity. }\end{array}$ & ० & $\bullet$ & ○ \\
\hline Q10 & $\begin{array}{l}\text { Forces between objects at the nanoscale arise when the objects are near each } \\
\text { other by chance. }\end{array}$ & $\bullet$ & $\circ$ & o \\
\hline Q11 & $\begin{array}{l}\text { Attractive forces between objects at the nanoscale are not important in } \\
\text { determining their behaviour. }\end{array}$ & o & $\bullet$ & ○ \\
\hline Q12 & $\begin{array}{l}\text { Forces between objects at the nanoscale are stronger when the surface contact } \\
\text { area between them is larger. }\end{array}$ & $\bullet$ & o & ○ \\
\hline Q13 & Nanotubes spontaneously aggregate together into rope-like structures. & $\bullet$ & o & o \\
\hline Q14 & $\begin{array}{l}\text { Attractive forces between objects at the nanoscale become weaker when the } \\
\text { contact surfaces of the objects have complementary shapes. }\end{array}$ & o & $\bullet$ & ○ \\
\hline Q15 & The smaller an object is, the smaller its surface area compared to its volume. & o & $\bullet$ & o \\
\hline Q16 & $\begin{array}{l}\text { The extremely high surface area to volume relationship of objects at the } \\
\text { nanoscale gives them different properties compared to everyday objects. }\end{array}$ & $\bullet$ & $\circ$ & ○ \\
\hline Q17 & $\begin{array}{l}\text { The physical properties (e.g. optical and electrical) exhibited by a substance do } \\
\text { not change when scaled up from the nanoscale to the size of everyday objects. }\end{array}$ & o & $\bullet$ & ○ \\
\hline Q18 & $\begin{array}{l}\text { Due to the size-dependent properties of matter, objects at the nanoscale can } \\
\text { exhibit functions not exhibited by everyday objects. }\end{array}$ & $\bullet$ & $\circ$ & ○ \\
\hline Q19 & $\begin{array}{l}\text { The unexpected behaviours and properties of objects at the nanoscale can be } \\
\text { exploited to develop useful technologies. }\end{array}$ & $\bullet$ & $\circ$ & ○ \\
\hline Q20 & $\begin{array}{l}\text { Nanotechnology allows scientists to arrange atoms, but only in ways that already } \\
\text { occur in nature. }\end{array}$ & o & $\bullet$ & ○ \\
\hline Q21 & $\begin{array}{l}\text { One branch of nanotechnology develops technologies by trying to mimic } \\
\text { naturally occurring biological structures. }\end{array}$ & $\bullet$ & $\circ$ & ○ \\
\hline Q22 & $\begin{array}{l}\text { Objects at the nanoscale (e.g. nanotubes) cannot be altered to adjust their } \\
\text { properties. }\end{array}$ & o & $\bullet$ & ○ \\
\hline Q23 & Nanotubes can be designed to bind to specific target cells inside the body. & $\bullet$ & ○ & ○ \\
\hline Q24 & $\begin{array}{l}\text { Modified nanotubes will move directly towards their intended target after being } \\
\text { introduced into the body. }\end{array}$ & 0 & $\bullet$ & 0 \\
\hline Q25 & $\begin{array}{l}\text { A modified nanotube that has attached to its specific target will remain } \\
\text { permanently bound. }\end{array}$ & 0 & $\bullet$ & 0 \\
\hline Q26 & $\begin{array}{l}\text { The properties of objects at the nanoscale may give rise to both advantages and } \\
\text { risks of nanotechnology. }\end{array}$ & $\bullet$ & 0 & 0 \\
\hline Q27 & $\begin{array}{l}\text { Nanosized objects (e.g. nanotubes) that have been inhaled cannot cause damage } \\
\text { to the lungs. }\end{array}$ & 0 & $\bullet$ & 0 \\
\hline Q28 & $\begin{array}{l}\text { Objects at the nanoscale (e.g. nanotubes) can be used in medical treatment of } \\
\text { diseases such as cancer. }\end{array}$ & $\bullet$ & 0 & 0 \\
\hline
\end{tabular}


Table 1 Overall demographic characteristics of respondents $(n=302)$ to the NanoKI

Demographic

Gender:

Female

139

Male

163

Age:

$$
\begin{aligned}
& \text { Mean } \\
& \text { Range }
\end{aligned}
$$

Level of education:

Primary

8

Upper secondary

30

Post-secondary $\leq 3$ years

56

Post-secondary $>3$ years

136

Graduate studies

Interest in nanoscience and nanotechnology:

Very uninterested

4

Uninterested

51

Interested

193

Very interested
38

13-79, median 36 
Table 2 Psychometric properties of the NanoKI validated with $n=302$

\begin{tabular}{|c|c|c|c|c|c|c|}
\hline Item & $\begin{array}{l}\text { Item difficulty } \\
\text { index }\end{array}$ & $\begin{array}{l}\text { Standard } \\
\text { Deviation }\end{array}$ & $\begin{array}{l}\text { Item discrimination } \\
\text { (D-value) }\end{array}$ & $\begin{array}{l}\text { Corrected item- } \\
\text { total correlation }\end{array}$ & $\begin{array}{l}\text { Cronbach's alpha } \\
\text { if item deleted }\end{array}$ & Factor loadings \\
\hline Q1 & 0.42 & 0.494 & 0.52 & 0.338 & 0.906 & 0.372 \\
\hline Q2 & 0.51 & 0.501 & 0.68 & 0.448 & 0.904 & 0.493 \\
\hline Q3 & 0.89 & 0.312 & 0.25 & 0.291 & 0.906 & 0.331 \\
\hline Q4 & 0.82 & 0.381 & 0.41 & 0.444 & 0.904 & 0.491 \\
\hline Q5 & 0.60 & 0.491 & 0.77 & 0.585 & 0.902 & 0.635 \\
\hline Q6 & 0.27 & 0.445 & 0.51 & 0.410 & 0.905 & 0.445 \\
\hline Q7 & 0.47 & 0.500 & 0.72 & 0.495 & 0.903 & 0.550 \\
\hline Q8 & 0.34 & 0.475 & 0.43 & 0.295 & 0.907 & 0.333 \\
\hline Q9 & 0.50 & 0.501 & 0.75 & 0.520 & 0.903 & 0.570 \\
\hline Q10 & 0.42 & 0.494 & 0.73 & 0.511 & 0.903 & 0.550 \\
\hline Q11 & 0.67 & 0.470 & 0.80 & 0.619 & 0.901 & 0.674 \\
\hline Q12 & 0.50 & 0.501 & 0.67 & 0.460 & 0.904 & 0.515 \\
\hline Q13 & 0.33 & 0.473 & 0.55 & 0.404 & 0.905 & 0.451 \\
\hline Q14 & 0.36 & 0.480 & 0.79 & 0.530 & 0.903 & 0.572 \\
\hline Q15 & 0.57 & 0.496 & 0.63 & 0.432 & 0.905 & 0.483 \\
\hline Q16 & 0.60 & 0.491 & 0.84 & 0.600 & 0.901 & 0.652 \\
\hline Q17 & 0.52 & 0.501 & 0.85 & 0.574 & 0.902 & 0.628 \\
\hline Q18 & 0.69 & 0.465 & 0.79 & 0.596 & 0.901 & 0.660 \\
\hline Q19 & 0.85 & 0.360 & 0.55 & 0.570 & 0.903 & 0.637 \\
\hline Q20 & 0.54 & 0.499 & 0.69 & 0.518 & 0.903 & 0.573 \\
\hline Q21 & 0.77 & 0.418 & 0.61 & 0.530 & 0.903 & 0.594 \\
\hline Q22 & 0.57 & 0.496 & 0.79 & 0.568 & 0.902 & 0.614 \\
\hline Q23 & 0.66 & 0.476 & 0.65 & 0.501 & 0.903 & 0.553 \\
\hline Q24 & 0.19 & 0.395 & 0.51 & 0.403 & 0.905 & 0.434 \\
\hline Q25 & 0.23 & 0.421 & 0.49 & 0.372 & 0.905 & 0.411 \\
\hline Q26 & 0.81 & 0.389 & 0.59 & 0.580 & 0.902 & 0.642 \\
\hline $\mathrm{Q} 27$ & 0.43 & 0.495 & 0.65 & 0.445 & 0.904 & 0.485 \\
\hline Q28 & 0.74 & 0.442 & 0.63 & 0.506 & 0.903 & 0.564 \\
\hline
\end{tabular}




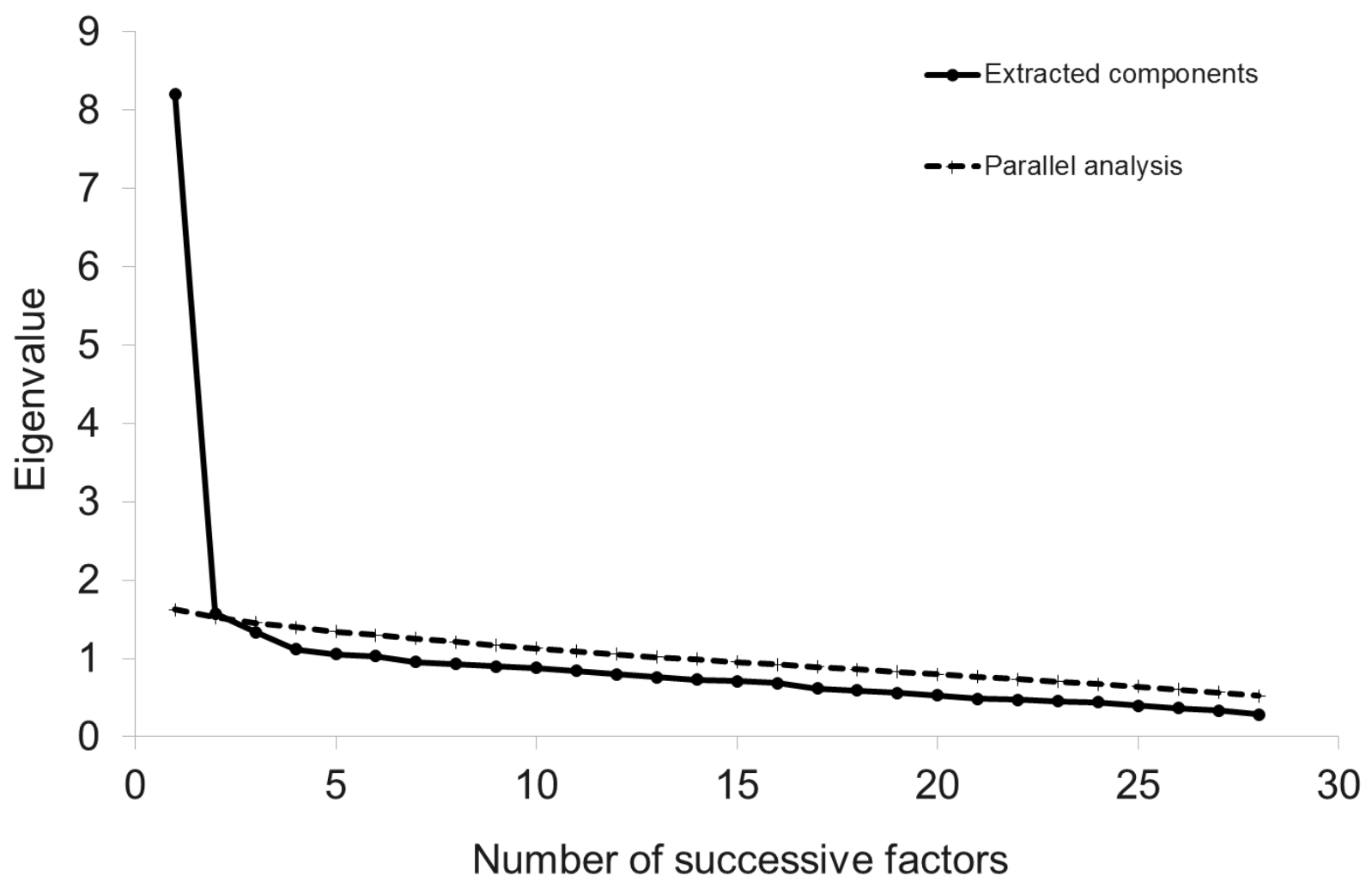

Fig. 1 Scree plot and parallel analysis used in the factor analysis of the data set $(28$ variables $\times 302$ respondents $)$ to determine the number of factors to retain. 